\title{
Comparison of demographic, and biochemical characteristics among younger and older patients with metabolic syndrome
}

\section{Fulden Sarac ${ }^{1}$, Pelin Tutuncuoglu ${ }^{2}$, Sumru Savas ${ }^{1}$, Sefa Sarac ${ }^{3}$, Fehmi Akcicek ${ }^{1}$ \\ ${ }^{1}$ Department of Geriatrics Medicine, Ege University Medical Faculty \\ ${ }^{2}$ Department of Endocrinology and Metabolism, Atatürk Training and Research Hospital \\ ${ }^{3}$ Department of Kardiology,Atatürk Training and Research Hospital}

Izmir/ TURKEY

- Aims: The metabolic syndrome (MetS) is a summary measure of important CVD risk factors that frequently coexist. The syndrome is evident in $20 \%$ to $30 \%$ of middle-aged women and has been linked to the development of CVD and diabetes. The aim of the study was to examine the differences in demographic and clinical profiles among older $(\geq 60$ years) and younger ( $<60$ years) patients with metabolic syndrome (MetS).

- $\quad$ Methods: The study was included 45 older (mean age $63.1 \pm 12.1$ yrs, 25 female,20 male ) and 40 younger (mean age $42.3 \pm 9.9 \mathrm{yrs}, 30$ female, 10 male) patients wih MetS. Metabolic syndrome (MS) was defined as in ATP III. Demographic and biochemical parameters were compared between groups. Insulin resistance (IR) was estimated using the homeostasis model assessment (HOMA).
- Results: Among older patients, $11.9 \%$ were smokers and $9.1 \%$ informed alcohol consumption. However, $29.5 \%$ of younger patients were smokers and only $12.1 \%$ of them consumed alcohol $(p=0.05, p=0.30)$. Mean levels of waist circumference, systolic, diastolic blood pressures, triglyceride and LDL-Cholesterol were statistically significantly higher than those of younger patients with MetS ( $p=0.001$, $\mathrm{p}=0.01, \mathrm{p}=0.001, \mathrm{p}=0.02$, $p=0.002$, respectively). Mean levels of HOMA-IR were found to be $2.3 \pm 0.9$ in young and $2.7 \pm 0.02$ in elderly group.

- Conclusions: There are differences between older and young patients with metabolic syndrome for waist circumference, smoking, blood pressures and lipid levels. 